\title{
OPTIMAL PRE-WEANING BODY WEIGHT AS AN EARLY SELECTION CRITERION FOR IMPROVING MEAT PRODUCTION IN BARKI SHEEP
}

\author{
El-Wakil, Salwa I.
}

Animal and Poultry Breeding Dept., Desert Research Centre, ElMatareya, Cairo, Egypt. Email: salwa_elwakil@yahoo.com

\section{SUMMARY}

The present study was initiated to investigate the importance of pre-weaning body weights in selection of Barki lambs. Four criteria were proposed to judge these pre-weaning body weights in order to assign the optimal one of them to be taken as an early selection criterion for improving meat production in Barki sheep. Pre-weaning body weight records and pedigree information of 2942 Barki lamb progenies of 186 sires and 1653 ewes belonging to the sheep flock of the Desert Research Centre raised in two research stations; Ras Elhekma (from 1963 to 1972) and Maryout (from 1973 to 2004) were used in the present study. The current study dealt with the lamb preweaning body weights from birth and at biweekly intervals till weaning at 120 days together with two adult body weights at 360 days and at 480 days. Data were analyzed by fitting a direct-maternal animal model using the MTDFREML program to estimate (co)variance components as well as direct and maternal heritabilities, correlations and breeding values for the studied traits.

Maternal heritabilities were higher than the corresponding direct ones from birth till the age of 30 days, and then turned to be lower for body weight at 45 days and up to weaning. Similar trends were revealed for maternal genetic variances and maternal breeding values compared with the corresponding direct ones. The estimated genetic correlations (either direct or maternal) were generally high and positive. The present study showed that body weight at 90 days, W90, as the "optimal" selection criterion to improve meat production in Barki sheep since it generally satisfies most of the criteria suggested to determine that optimal body weight. Compared with the other pre-weaning body weights, W90 had generally higher heritability and breeding values. W90 had also higher utility in predicting adult body weights through high and positive direct and maternal genetic and environmental correlations. Higher genetic, phenotypic and environmental variation occurred in W90. There is no antagonism relationship between direct and maternal effects existed for $W 90$ and between this trait and adult body weights. Therefore, it is anticipated that higher genetic response would be attained if the selection based on W90. Accordingly, the present study recommends W90 as the optimal early selection criterion for breeding purposes aiming at genetic improvement for meat production in Barki sheep.

Keywords: pre-weaning body weights, genetic parameters, early selection, Barki sheep

\section{INTRODUCTION}

Genetic aspects of body weights become vital for breeders and heavier body weights have been the primary selection emphasis for meat production in many breeds. There is no published information on body weight records at biweekly intervals during the pre-weaning stage (except for birth and weaning weights) in Barki sheep; one of the main indigenous breeds of Egypt dominated the north western coastal belt. Knowledge of genetic variations and correlations between traits are important to develop appropriate breeding programs aimed to maximizing genetic improvement. Studies of various breeds as well as Barki sheep have shown that body weight is influenced by direct genetic effects in addition to the maternal genetic and environmental components and both effects should be taken into account in the breeding program to achieve adequate genetic progress (Ligda et al., 2000 and Ghafouri-Kesbi and Baneh, 2012). The impact of maternal genetic and environmental effects in controlling body weight and growth performance in Barki sheep was obvious from birth up to the yearling stage (El-Wakil and
Gad, 2014). Furthermore, the present study employed the pre-weaning body weights of Barki sheep to define the optimal pre-weaning body weight to be taken as an early selection criterion for improving meat production. Four criteria were proposed to judge that optimal body weight among other pre-weaning body weights. The genetic contribution of such body weight should be emphasized since the selection will be based on this trait. The genetic and phenotypic variation should also be considered since the higher the variability the higher the efficiency of selection and hence the more genetic gain would be. The utility of such body weight to predicting adult body weights would be of an advantage. That optimal body weight would be favourable if the positive associations between direct and maternal genetic effects are existed within and between the concerned traits to ensure adequate genetic progress. The present study provided such information to examine these four criteria for the pre-weaning body weights in order to help reach an overall decision for the optimal body weight to be 
used for breeding purposes. Therefore, the current study has two objectives; firstly to thoroughly evaluate the pre-weaning body weights by producing various direct and maternal genetic parameters as prerequisites to develop breeding strategies for Barki sheep, and secondly to assign the optimal preweaning body weight to be considered as an early selection criterion to improve meat production, and hence profitability and sustainability of the native Barki sheep.

\section{MATERIALS AND METHODS}

\section{Data:}

Pre-weaning body weight records and pedigree information of Barki lambs were originated from the sheep flock of the Desert Research Centre maintained at two research stations; Ras Elhekma (from 1963 to 1972) and Maryout (from 1973 to 2004). Management of the flock was almost the same where ewes were often first mated at approximately 16 months of age and being subjected to culling unless they are suitable for breeding purposes. The breeding season usually carried out once a year in June - July to start lambing in October - November. At birth, lambs were weighed to assign birth weight, then body weights were recorded at biweekly intervals till weaning followed by monthly intervals till the animal removed from the flock. Shearing took place once a year during April- May. Feeding of the flock depends mainly on grazing at Ras Elhekma while rely on cut and carry at Maryout research station. Detailed flock management was described by El-Wakil et al. (2009).

The present study dealt with pre-weaning body weights from birth and at biweekly intervals up to weaning at approximately four months of age. Two adult body weights were also recorded after one year of age, 360 days (W360) and at the first mating season at 16 months, 480 days (W480). Birth weight, $B W$, was kept as recorded while all other studied body weights were linearly adjusted to body weights at 15 days, W15, at 30 days, W30, at 45 days, W45, at 60 days, $W 60$, at 75 days, $W 75$, at 90 days, $W 90$, at 105 days, W105 and at weaning, 120 days, W120 as well as W360 and W480, respectively. The adjustments for individual body weights to different ages were made by interpolation between the data of two successive ages assuming linear growth function during the short intervals. Editing of the original data was done to remove the few numbers of twinning from the data set together with those animals that had missing identifications. After editing, the data set consisted of 2942 records of lambs' progenies of 186 sires and 1653 ewes. The number of records for the studied pre-weaning body weights are shown in Table (1) while the number, average $\pm \mathrm{SE}$ and coefficient of variation were also estimated for $W 360(1716,31.57 \pm 0.18 \mathrm{~kg}$ and $22.9 \%)$ and $W 480$ (1474 and $36.63 \pm 0.20 \mathrm{~kg}$ and $20.6 \%$ ).

\section{Statistical Analysis}

Using the general linear model (GLM) procedure of SAS (2004), preliminary analyses were conducted and indicated that lamb gender, year of birth, dam age and location are significant fixed effects for the studied traits. Consequently, they were introduced for further analysis by fitting an animal model using the MTDFREML program (Boldman et al., 1995) to estimate (co) variance components and the corresponding genetic parameters as well as expected breeding values. While there is a series of models including various combinations of maternal and direct effects could be fitted, the following most parameterized direct-maternal model was used to calculate the required genetic parameters. The model included the same fixed effects mentioned earlier and considering the animal, sire and dam as random effects as follows:

$\mathbf{Y}=\mathbf{X b}+\mathbf{Z}_{\mathrm{a}} \mathbf{a}+\mathbf{Z}_{\mathrm{m}} \mathbf{m}+\mathbf{Z}_{\mathrm{c}} \mathbf{c}+\mathbf{e}$, where

$\operatorname{Cov}(\mathbf{a}, \mathbf{m})=\mathbf{A} \sigma_{\mathrm{am}}$

where $Y$ is a vector of observations of the studied traits; b, a, m, c and e are vectors of fixed effects, direct additive genetic effects, maternal additive genetic effects, maternal permanent environmental effects and the residual effects, respectively. $\mathrm{X}, \mathrm{Z}_{\mathrm{a}}, \mathrm{Z}_{\mathrm{m}}$ and $\mathrm{Z}_{\mathrm{c}}$ are incidence matrices relating observations to the fixed effects, direct additive genetic effects, maternal additive genetic effects and maternal permanent environmental effects, respectively. It was assumed that direct additive genetic, maternal additive genetic, maternal permanent environmental and residual effects to be normally distributed with mean of zero and variance of $A \sigma_{a}^{2}, A \sigma_{m}^{2}, I_{d} \sigma^{2} c$ and $I_{n} \sigma_{e}^{2}$, respectively; where, $\sigma_{a}^{2}, \sigma_{m}^{2}, \sigma_{c}^{2}$ and $\sigma_{e}^{2}$ are direct additive genetic variance, maternal additive genetic variance, maternal permanent environmental variance and the residual variance, respectively. " $A$ " is the additive numerator relationship matrix, while $I_{d}$ and $I_{n}$ are identity matrices with dimensions equal to the number of the dams and number of records, respectively. Moreover, $\sigma_{a m}$ refers to the covariance between direct additive genetic and maternal additive genetic effects.

(Co) variance components were used to estimate heritabilities and correlation coefficients for the studied traits. Direct $\left(\mathrm{h}_{\mathrm{a}}^{2}=\sigma_{a}^{2} / \sigma_{p}^{2}\right)$ and maternal heritabilities $\left(\mathrm{h}_{\mathrm{m}}^{2}=\sigma_{m}^{2} / \sigma_{p}^{2}\right)$ were done using a series of univariate analyses for each studied trait, where $\sigma_{p}^{2}$ is the phenotypic variance. Error variance was estimated directly from the residual sums of squares. Estimates of correlation coefficients between each of the pre-weaning body weight and both W360 and W480 were done using a series of bivariate analyses. The appropriate covariance, phenotypic or genetic, between each pair of studied traits, $\mathrm{x}$ and $\mathrm{y}$, was divided by the square root of the product of the corresponding variance of each trait (rxy $=\sigma x y / \sqrt{ }$ $\left(\sigma^{2} \mathrm{x} * \sigma^{2} \mathrm{y}\right)$. The maternal genetic, maternal permanent environmental and direct environmental correlations were calculated in the same manner. For 
the pre-weaning body weights, the direct-maternal genetic correlation $\left(r_{a m}\right)$ was computed as the ratio of the direct-maternal genetic covariance $\left(\sigma_{a m}\right)$ to the product of the square roots of $\sigma^{2}{ }_{a}$ and $\sigma_{m}^{2}$ within the same trait. On the other hand, cross-correlations between direct genetic effects for one trait and maternal genetic effects for another trait (denoted as $\mathrm{r}_{\mathrm{a} 1 \mathrm{~m} 2}$ ) were calculated as the average of $\mathrm{r}_{\mathrm{a} 1 \mathrm{~m} 2}$ and $\mathrm{r}_{\mathrm{a} 2 \mathrm{~m} 1}$. Direct (DBVs) and maternal breeding values (MBVs) of individual animals were estimated using the direct-maternal animal model previously mentioned through MTDFREML software (Boldman et al., 1995). Consequently, the mean values of DBVs and MBVs for each studied trait were calculated using SAS computer package (SAS, 2004).

\section{RESULTS AND DISCUSSION}

There is a trend for the least square means as well as phenotypic $\left(\sigma_{p}^{2}\right)$, direct $\left(\sigma_{\mathrm{a}}^{2}\right)$, maternal $\left(\sigma_{m}^{2}\right)$, environmental $\left(\sigma_{e}^{2}\right)$ and permanent environmental $\left(\sigma_{c}^{2}\right)$ variances to generally increase as age advanced during the pre-weaning stage. Birth weight seems to have the least variations in terms of standard errors and coefficient of variation as well as $\sigma_{p}^{2}, \sigma_{\mathrm{a}}^{2}, \sigma_{\mathrm{m}}^{2}$, $\sigma_{\mathrm{e}}^{2}$ and $\sigma_{\mathrm{c}}^{2}$ compared with other studied body weights (Table 1). Barki sheep are usually bred under harsh environmental conditions which created wide range of genetic and phenotypic variation for the studied pre-weaning body weights. Estimates of $\sigma_{\text {e }}^{2}$ were consistently higher than the corresponding $\sigma^{2}$ for the studied traits, bearing in mind that $\sigma^{2}{ }_{\mathrm{e}}$ includes all unaccounted for sources of variation, not only "true environmental" effects. The amounts of genetic variability (direct and maternal) exhibited for the studied pre-weaning body weights suggest the possibility of enhancing the genetic gain for these traits.

Table (1) shows that direct $\left(h^{2}\right)$ and maternal heritability $\left(h_{m}^{2}\right)$ for pre-weaning body weights ranged from 0.07 to 0.18 and from 0.07 to 0.19 , respectively which revealed that selection for improved maternal and/or direct genetic effects would generate slow to moderate genetic progress in such earlier body weights of Barki lambs. The highest maternal heritability was obtained for birth weight (0.19) and tends to generally decline towards weaning. $h_{m}^{2}$ were higher than $h^{2}$ from birth till W30, and then turned to be lower from W45 up to weaning. As expected, similar trend existed for maternal genetic variances $\left(\sigma_{m}^{2}\right)$ and maternal breeding values $(M B V)$ compared with direct genetic variance $\left(\sigma_{a}^{2}\right)$ and direct breeding values $(D B V)$ as these are functions of each other. On the other hand, the estimated $D B V$ and $M B V$ for the studied pre-weaning body weights showed slight differences which probably indicate similar impacts of both estimates in controlling these traits and hence genetic improvement for growth performance in Barki sheep must depend entirely on both direct and maternal breeding values.
Data in Table (1) show that genetic covariances $\left(\sigma_{a m}\right)$ and correlations $\left(r_{a m}\right)$ between direct and maternal genetic effects within each studied trait had similar trend in which they were all zeros while positive for $B W$ and negative for W60, W105 and W120. On the other hand, correlations $\left(r_{\text {alm2 }}\right)$ between direct and maternal genetic effects for each studied pre-weaning body weight with adult body weights (W360 and W480) were all positive except those negative correlations for W45 with W480 (0.12 ) and W120 with W360 (-0.21). In case of negative correlations between direct and maternal genetic effects in given traits, further selection of animals by considering only direct genetic effects could mask true genetic potential of lambs and decline the maternal ability for these traits. Care must be taken to minimize the impact of negative correlations between direct and maternal genetic effects within and between target traits in order to improve the genetic progress in the flock which could be done through an appropriate balance between these two types of effects. Similar findings were reported in the literature (Tosh and Kemp, 1994 and Supakorn et al., 2013).

Table (1) presents correlation coefficients of the studied pre-weaning body weights with W360 and W480 regarding the direct genetic, $r_{\text {ala2 }}$, maternal genetic, $r_{m 1 m 2}$, direct environmental, $r_{\text {ele2 }}$ and maternal permanent environmental correlations, $r_{c 1 c 2}$. While all these correlations were generally positive, there is a few negative associations existed for birth weight $\left(r_{c l c 2}\right.$ with W360 and W480), W15 ( $r_{c 1 c 2}$ with W480), W45 $\left(r_{m 1 m 2}\right.$ with W480), W75 ( $r_{\text {ala2 }}$ with W480 and $r_{c l c 2}$ with W480), W105 ( $r_{a 1 a 2}$ with W480) and W120 ( $r_{\text {ala2 }}$ with W360). The estimated genetic correlations (either direct or maternal) were generally high and positive. Genes with similar effects and / or genes with pleiotropic effects could be operating on these traits. Moreover, in case of direct genetic correlations obtained between W75 and W105 with W360 and $W 480$, there is a difference in which one is high positive and the other is high negative in each case. While it could be accepted that pre-weaning growth measured at any stage is influenced by the same set of genes, in some cases the expression of genes might be changed from one stage to another one. Apart from these few negative correlations, high and positive genetic correlations obtained, generally revealed that selection for such pre-weaning body weights based on genetic correlation might have positive effect for increased adult body weight and could contribute indirectly to improve lamb survivability (Ahunu et al., 1997; Ligda et al., 2000; Van Vleck et al., 2003 and Szwaczkowski et al., 2006). On the other hand, maternal correlation coefficients (either genetic or environmental) seemed to be generally high and positive. The maternal additive genetic and environmental effects, which favor the growth of fetus, could also have some beneficial effect on post-natal growth traits. Similar explanations were reported elsewhere (Maria et al., 1993; Gowane et al., 2010; Rashidi, 2012; Ghafouri-Kesbi and Baneh, 2012 and 
El-Wakil and Gad, 2014). As far as the environmental correlations (direct and maternal) are concerned, both $r_{c 1 c 2}$ and $r_{e l e 2}$ ranged from moderate to high, the former being generally higher than the latter. Direct environmental correlations of the studied pre-weaning body weights with adult body weights were consistently positive. The respective maternal permanent environmental correlations were generally high and positive demonstrating the importance of maternal permanent environment to control the studied pre-weaning and adult body weights. However, such maternal permanent environment seemed to be negative with adult body weights in terms of the correlations $\left(r_{c 1 c 2}\right)$ of birth weight (with $W 360$ and W480), W15 (with W480) and W75 (with W480). Maternal environmental effects on body weights could possibly be determined by uterine capacity, feeding level during late gestation and maternal behavior of the ewe during suckling (Gowane et al., 2010; Thiruvenkadan et al., 2011 and Shokrollahi and Baneh, 2012). Similar findings were reported in Barki sheep of this flock in which maternal influences tended to sustain throughout the first year from birth to weaning up to yearling body weights (El-Wakil and Gad, 2014).

Table (1). Genetic, phenotypic and environmental parameters for pre-weaning body weights at birth (BW), 15 days (W15), 30 days (W30), 45 days (W45), 60 days (W60), 75 days (W75), 90 days (W90), 105 days (W105) and 120 days (W120), 360 days (W360) and 480 days (W480) in Barki sheep

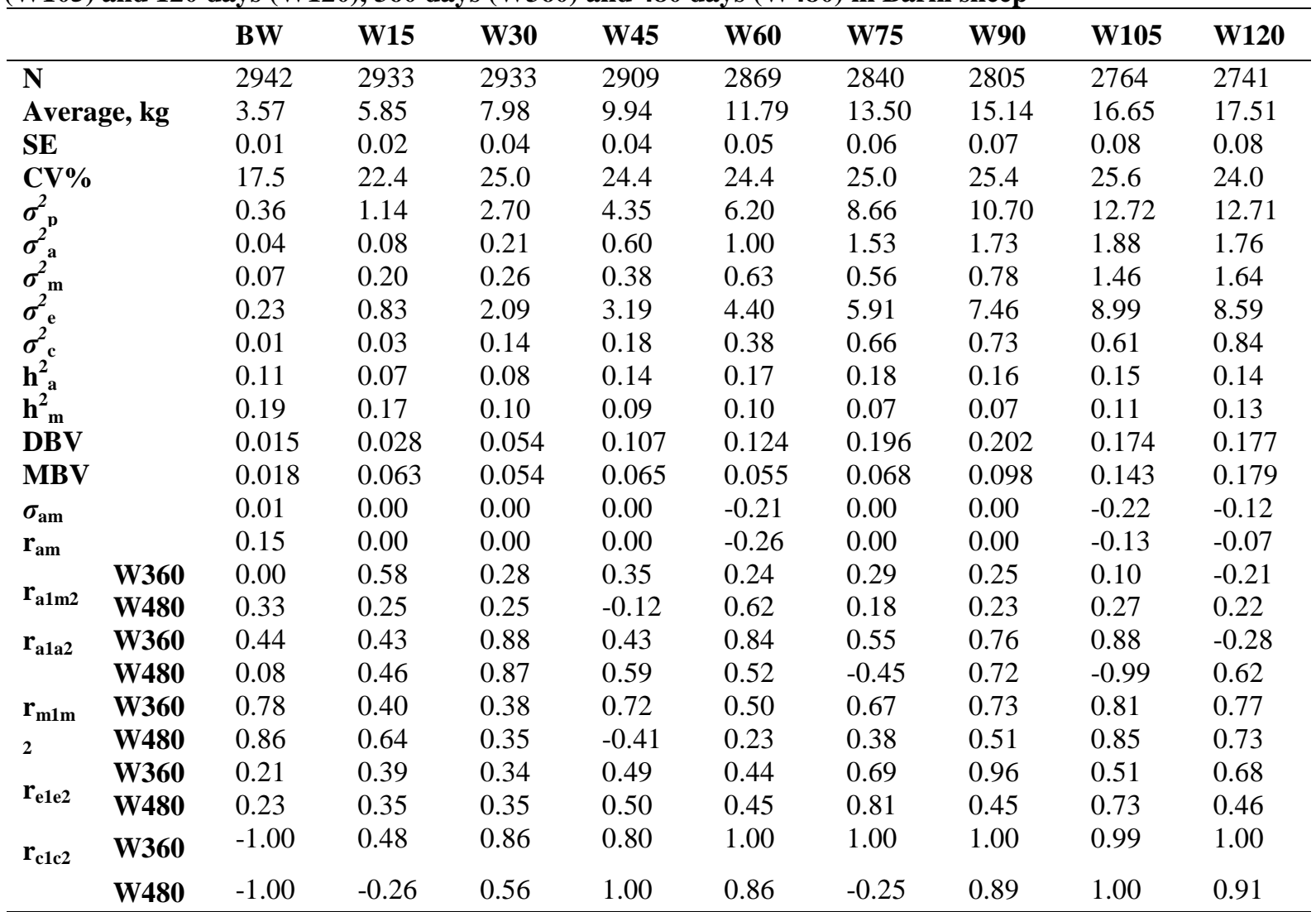

$\boldsymbol{S E}=$ standard errors, $\boldsymbol{C} \boldsymbol{V} \%=$ coefficient of variation, $\sigma_{p}^{2}=$ phenotypic variance, $\boldsymbol{\sigma}_{a}^{2}=$ additive genetic variance, $\sigma_{m}^{2}=$ maternal genetic variance, $\sigma_{e}^{2}=$ residual variance, $\sigma_{c}^{2}=$ maternal permanent environmental variances, $\boldsymbol{h}_{a}^{2}=$ direct heritability, $\boldsymbol{h}^{2}{ }_{\boldsymbol{m}}=$ maternal heritability, $\boldsymbol{D} \boldsymbol{B} \boldsymbol{V}=$ direct breeding value, $\boldsymbol{M B} \boldsymbol{V}=$ maternal breeding value, $\boldsymbol{\sigma}_{\boldsymbol{a m}}=$ direct- maternal genetic covariance within a trait, $\boldsymbol{r}_{\boldsymbol{a m}}=$ direct- maternal genetic correlation within a trait, $\boldsymbol{r}_{\boldsymbol{a l m} \mathbf{2}}=$ direct- maternal genetic correlation between two traits, $\boldsymbol{r}_{\boldsymbol{a l a 2}}=$ direct genetic correlations, $\boldsymbol{r}_{\boldsymbol{m} \mathbf{m} \mathbf{2}}=$ maternal genetic correlations, $\boldsymbol{r}_{\boldsymbol{e l e} 2}=$ direct environmental correlations, and $\boldsymbol{r}_{\boldsymbol{c l c 2}}=$ maternal permanent environmental correlations.

\section{Optimal pre-weaning body weight}

One of the objectives of the current study was to define an "optimal" pre-weaning body weight to be taken as an early selection criterion for improving meat production in Barki sheep. Therefore, the following four criteria were suggested to reach an overall decision for that "optimal" live body weight:

1. High genetic contribution (direct and maternal heritabilities and breeding values) since the selection will be based on this trait,

2. High genetic and phenotypic variation, since the higher the variability the higher the efficiency of selection and hence more genetic gain would be,

3. High ability to aid in early selection for adult body weights (W360 and W480) in terms of direct and maternal genetic and environmental correlations,

4. No antagonism relationships between direct and maternal genetic effects, both within and between target traits.

Consequently, a comparison has to be made among the studied pre-weaning body weights against these four criteria. For each pre-weaning body weight, this "optimal" body weight can be picked for each suggested criterion, and then a comparison among all 
pre-weaning body weights could be done to choose that body weight which in general satisfies most of these criteria.

Table (1) exhibited negative correlations $\left(r_{a m}\right)$ and covariances $\left(\sigma_{a m}\right)$ between direct and maternal genetic effects within each of W60,W105 and W120. There is also negative direct-maternal genetic correlation between $W 45$ and $W 480\left(r_{a 1 m 2}\right.$ of -0.12$)$. These antagonism relationships between direct and maternal effects indicate that selection for improved maternal and/or direct effects is expected to generate only slow genetic progress in terms of W45, W60, W105 and W120. Moreover, maternal genetic correlation was found to be moderate and negative (0.41 ) between W45 and W480 which would inferior the maternal performance and resulted in reducing selection response for these body weights. Accordingly, W45, W60, W105 and W120 might not be optimal body weights for improving meat production in Barki sheep.

Birth weight showed the highest maternal heritability (0.19) compared with other pre-weaning body weights. That is probably matched with the biological perspective, that birth weight is strongly determined by maternal conditions during embryological development (Ahunu et al., 1997; Ligda et al., 2000; Van Vleck et al., 2003 and Szwaczkowski et al., 2006). Hence, birth weight is affected more by the potentiality of the dam rather than by the intrinsic potentiality of the lamb itself. However, birth weight had low estimate of direct heritability (0.11) together with the least estimates of phenotypic, direct and maternal genetic variances as well as least direct and maternal breeding values which indicate that a slow genetic progress is expected from the selection for this trait. Furthermore, positive correlation between direct and maternal genetic effects within and between traits $\left(r_{a m}\right.$ and $\left.r_{a l m 2}\right)$ together with the positive direct and maternal genetic correlations between birth weight and later body weights may account for increasing lamb birth weight and possible lambing difficulties. It is also reported that selection on the basis of birth weight could cause dystocia in Bakhtiari sheep of Iran (Edriss et al., 2002). Thus, birth weight probably not a suitable selection criterion since it might not afford high genetic response and to avoid those restrictions affecting lamb survivability.

As far as the remaining pre-weaning body weights (W15, W30,W75 and W90), it appeared that W75 had the highest direct heritability (0.18), however, negative direct genetic correlation of -0.45 between $W 75$ and $W 480$ threaten the predictability of $W 75$ to the later body weights.

Body weight at 90 days (W90) showed the highest phenotypic as well as direct and maternal genetic and environmental variability among the remaining studied pre-weaning traits. Relatively high genetic variability (direct and maternal) estimated for W90 suggest that Barki sheep could quickly respond to selection programs to improve this trait and subsequent body weights. Although
W90 has low maternal heritability (0.07), it showed the highest direct heritability (0.16). The highest direct (0.202) and maternal breeding values (0.098) obtained for W90 is of advantage to W90 compared with other pre-weaning body weights since the selection is usually based on breeding values to ensure high genetic gain. Moreover, there is no negative association between direct and maternal genetic effects exhibited for $W 90$ and between this trait and adult body weights which indicate the possibility of improving direct and/ or maternal effects through selection for $W 90$ to attain adequate genetic progress.

As far as the correlations with the adult body weights are concerned, W90 showed high and positive direct $(0.76$ and 0.72$)$ and maternal genetic correlations (0.73 and 0.51$)$ with W360 and W480, respectively. This result might indicate that $W 90$ is an effective early selection criterion and able to genetically change the adult body weights and hence improve meat production in Barki sheep. Good management conditions and favorable maternal behavior would also have a positive impact on $W 90$ of lambs and subsequent body weights as a result of direct (0.96 and 0.45) and maternal permanent environmental correlations (1.00 and 0.89) with $W 360$ and $W 480$, respectively. It appeared that W90 is positively associated with heavier adult body weights and could also contribute indirectly to improve lamb survivability. From the sheep husbandry point of view, this result could be of beneficial to save time for the breeder to allow recording lamb's body weights only at 90 days during the pre-weaning stage.

\section{CONCLUSION}

Lamb' body weight at 90 days, W90, could be considered as the optimal body weight since it is shown to be heritable with the highest breeding values, consequently it is favored and higher genetic response is expected if the selection based on this trait. W90 gave the best utility to aid in early selection for adult body weights. Large amounts of genetic, phenotypic and environmental variation occurred in W90. It is also more satisfactory since there is no antagonism relationship between direct and maternal effects existed for $W 90$ and between this trait and later body weights. So, it is rational to recommend body weight at 90 days as an early selection criterion to be included in the breeding scheme for improving meat production in Barki sheep.

\section{ACKNOWLEDGEMENT}

Prof. Hassanein El-Gabbas is greatly acknowledged for his valuable comments and reading the manuscript.

\section{REFERENCES}


Ahunu B. K., P. F. Arthur and H. W. A. Kissiedu, 1997. Genetic and phenotypic parameters for birth and weaning weights of purebred and crossbred Ndama and West African Shorthorn cattle. Livestock Production Science, 51:165171.

Boldman K. G., L. A. Kriese, L. D. Van Vleck and C. P. Van Tassell, 1995. A manual for use of MTDFREML. A set of programs to obtain estimates of variances and covariances. ARS, USDA, Washington.

Edriss M. A., F. Tahmasebi and M. Vatan-Khah, 2002. Estimation of variance components for pre-weaning weights in Bakhtiari sheep breed. Proceedings of $7^{\text {th }}$ World Congress on Gentics Applied to Livestock Production, Montpellier, France.

El-Wakil Salwa I., Manal Elsayed, A. M. Ahmed, R. R. Sadek and A. A. Nigm, 2009. Genetic and phenotypic parameters of birth, weaning and yearling body weights of Barki sheep raised in the north western coast of Egypt. Egyptian J. Anim. Prod., 46 (1): 43-52.

El-Wakil Salwa I. and S. M. A. Gad, 2014. Evaluation of direct and maternal (co) variance components and heritabilities for some body weights and growth traits in Barki sheep. Egyptian Journal of Sheep and Goat Sciences, 9 (1): 21- 30.

Ghafouri-Kesbi F. and H. Baneh, 2012. Genetic parameters for direct and maternal effects on growth traits of sheep. Archiv Tierzucht, 55 (6): 603-611.

Gowane G. R., A. Chopra, V. Prakash and A. L. Arora, 2010. Estimates of (co) variance components and genetic parameters for body weights and first greasy fleece weight in Malpura sheep. Livestock Science, 131: 94-101.

Ligda Ch., G. Gabriilidisb, Th. Papadopoulosb and A. Georgoudisa, 2000. Investigation of direct and maternal genetic effects on birth and weaning weight of Chios lambs. Livestock Production Science, 67: 75-80.

Maria G. A., K. G. Boldman and L. D. Van Vleck, 1993. Estimates of variances due to direct and maternal effects for growth traits of Romanov sheep. Journal Animal Science, 71:845-849.

Rashidi A., 2012. Genetic parameter estimates of body weight traits in Iran-Black sheep. Journal of Livestock Science and Technologies, 1 (1): 5460.

SAS 2004. STAT Software User's Guide, version 9.0, SAS Institute, Cary, NC, USA.

Shokrollahi B. and H. Baneh, 2012. (Co) variance components and genetic parameters for growth traits in Arabi sheep using different animal models. Genetics and Molecular Research, 11(1): 305-314.

Supakorn C., Winai Pralomkarn, Suwit Anothaisinthawee, 2013. Estimation of genetic parameters and genetic trends for weight and body measurements at birth in sheep populations in Thailand. Songklanakarin J. Sci. Technol., 35 (1): 1-10.

Szwaczkowski T., Wójtowski, J., Stanisławska, E., Gut, A., 2006. Estimates of maternal genetic and permanent environmental effects in sheep. Archiv Tierzucht, 49: 186-192.

Thiruvenkadan A. K., K. Karunanithi, J. Muralidharan and R. Narendra Babu, 2011. Genetic analysis of pre-weaning and postweaning growth traits of Mecheri sheep under dry land farming conditions. Asian-Aust. Journal of Animal Science, 24(8):1041-1047.

Tosh J. J. and R. A. Kemp, 1994. Estimation of variance components for lamb weights in three sheep populations. Journal of Animal Science, 72: 1184-1190.

Van Vleck L. D., G. D. Snowder and K. J. Hanford, 2003. Models with cytoplasmic effects for birth, weaning, and fleece weights and litter size at birth for a population of Targhee sheep. Journal Animal Science, 81: 61-67. 


\section{وزن الجسم الأمثل فى مرحلة ما قبل الفطام كوسيلة للإنتخاب المبكر لتحسين إنتاج اللحم فى الأغنام البرقى}

سلوى إبراهيم الوكيل قسم تربية الحيوان والدواجن ، مركز بحوث الصحراء، المطرية، القاهرة، مصر

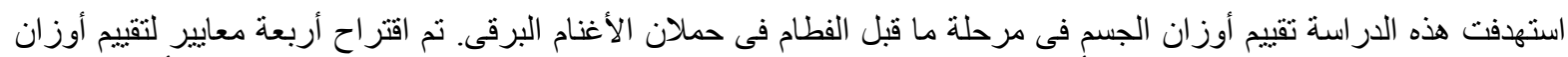

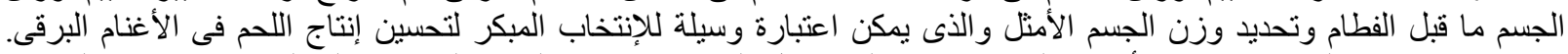

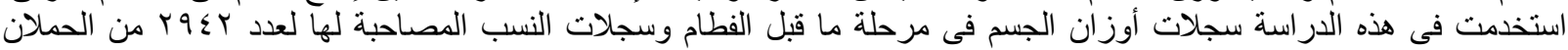

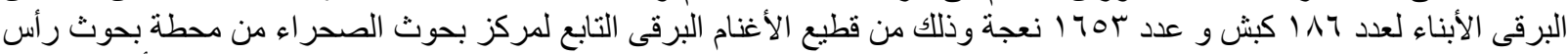

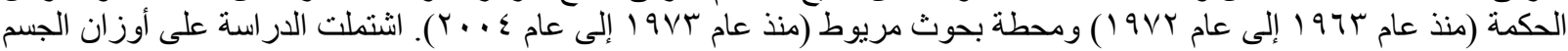

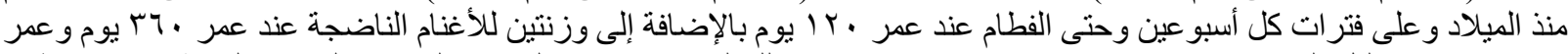

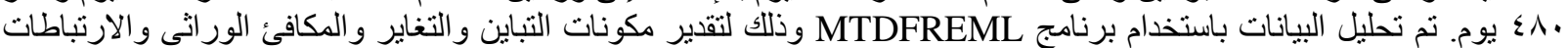
و القيم التربوية للصنفات المدروسة.

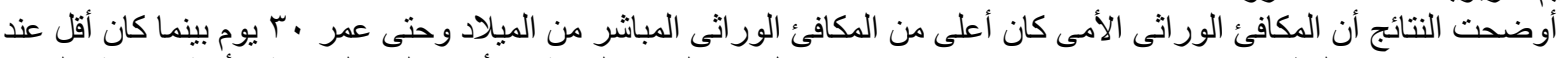

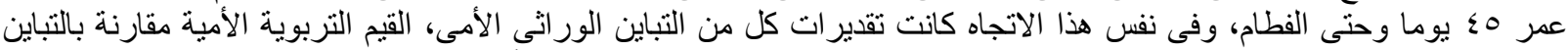

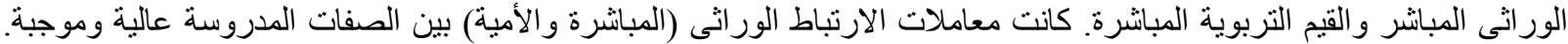

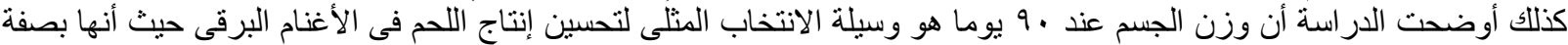

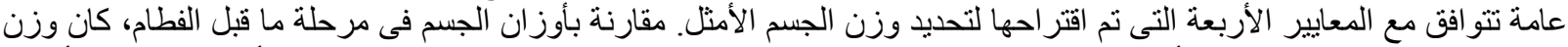

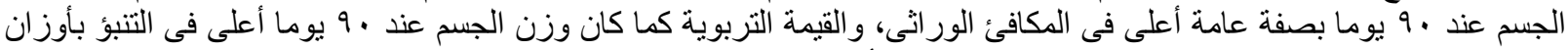

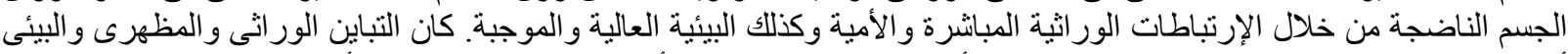

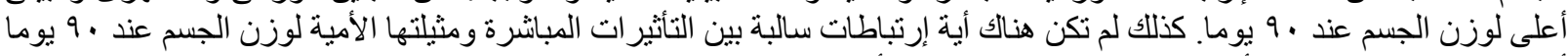

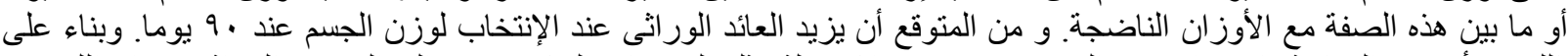

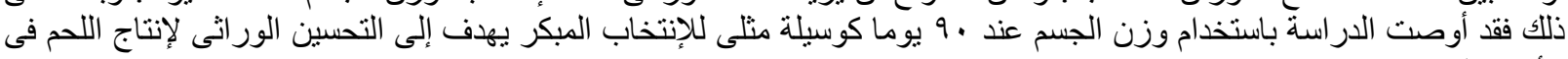

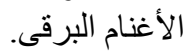

